Ann. Zootech., I968, $17(4), 429-443$.

\title{
GLYCOGÉNOLYSE POST MORTEM DU MUSCLE LONGISSIMUS DORSI DE PORC
}

\author{
J. CHARPENTIER (1) \\ ivec la collaboration technique de Anne-Marie Guevgneau et P. Poterre \\ Laboratoire de Recherches sur la Viande, \\ Centre national de Recherches zootechniques, 78 -Jouy-en-Josas \\ Institut national de la Recherche agronomique
}

\section{SOMMAIRE}

Il est bien établi que la vitesse de chute du pH post mortem des muscles de porc est très variable et qu'elle influence directement de nombreux aspacts de la qualité de la viande. Aussi, la présente étude consiste en une étude descriptive de la glycogénolyse post mortem du muscle de porc afin d'interpréter, si possible, les différences de rapidité de cette glycogénolyse. Nous avons utilisé zo porcs de race Large While et 20 porcs de race Piétrain d'un poids compris entre roo et I Io kgs.

Les dosages étaient effectués périodiquement sur une partie de Longissimus dorsi prélevée immédiatement à l'abattage et maintenue sous azote à $30^{\circ} \mathrm{C}$ pendant une durée de 240 minutes. Les muscles étaient classés en 2 groupes en fonction de la valeur $\mathrm{du}$ " $\mathrm{pH} 40$ minutes ". Lorsque le " $\mathrm{pH}$ 40 minutes " était supérieur à 6,00 , la diminution du $\mathrm{pH}$ était considérée comme lente (groupe $\mathrm{L}$ ), alors qu'elle était considérée comme rapide (groupe $\mathrm{R}$ ), lorsque le $\mathrm{pH}$ était inférieur à 6,00. Le groupe L comprenait 14 Large White et 4 Piétrains. Le groupe $\mathrm{R}$ comprenait 16 Piétrains et 6 Large White.

Nous avons déterminé le glycogène, le gluco ie, les sucres réducteurs totaux, l'acide lactique, les substrats intermédiaires de la glycogénolyse, l'ATP, l'ADP, la phosphocréatine, le phosphate minéral, le phosphate acidosoluble total, le $\mathbf{P}$ " o minute ", le $\mathbf{P}$ " 7 minutes". Les résultats de cette étude montrent des différences de teneurs initiales en ce qui concerne principalement la phosphocréatine $(R$ :

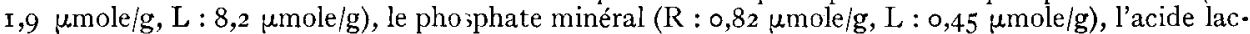
tique $(\mathrm{R}: 64,2 \mu$ moles $/ \mathrm{g}, \mathrm{L}: 26,3$ umoles $/ \mathrm{g}$ ). La faible variabilité des substrats intermédiaires indique que des modifications d'activité des enzymes de la glycogénolyse ne sont vraisemblablement pas à l'origine des différences de vitesse de la glycogénolyse post mortem. La différence principale entre les muscles des groupes $\mathrm{R}$ et $\mathrm{L}$ réside dans l'activité ATPasique. Les activités ATPasiques sont en effet en moyenne de $0,16 \mu$ mole d'ATP dégradé $/ \mathrm{g} / \mathrm{mn}$ pour le groupe $\mathrm{L}$ et de $0,38 \mu / \mathrm{mole}$ d'ATP/g/mn pour le groupe R. Le problème essentiel consiste donc à élucider les mécanismes responsables de la régulation de l'activité ATPasique post mortem.

\section{INTRODUCTION}

A l'issue des travaux de nombreux auteurs (WISMER-PEDERSEN, LAWRIE, I96o; WisMER-PEDERSEN et Briskey, I96I ; BENDALL et al., ig62) il est actuellement bien établi que la rapidité de la diminution du $\mathrm{pH}$ musculaire après la mort, conditionne

(1) Adresse actuelle : Laboratoire de Recherches sur la Viande CRVZ -63 - Theix par St GENESCHAMPANELLE.

Annales de Zootechnie. - ig68. 
en grande partie diverses caractéristiques physico-chimiques responsables de la qualité des viandes. Par suite d'une glycogénolyse particulièrement rapide, il se peut en effet que le $\mathrm{pH}$ musculaire atteigne des valeurs relativement faibles alors que la température est voisine de celle de l'animal vivant. Une telle conjonction $\mathrm{pH}$-température est à l'origine d'une dénaturation des protéines sarcoplasmiques (KRzYWICKI, I962; MC LOUGHLIN, I963; SCOPES, I964) et vraisemblablement des protéines myofibrillaires également (PENNY, 1967). La dénaturation de ces protéines modifie diverses caractéristiques du tissu musculaire dont en particulier la coloration et l'aptitude à la rétention d'eau. Il semble que ce soit dans l'espèce porcine que l'évolution post mortem du pH musculaire présente la plus grande variabilité et que, par conséquent, ses incidences sur le plan technique soient les plus importantes. Bien que depuis plusieurs années de nombreux travaux aient été consacrés à l'étude de divers facteurs susceptibles d'être impliqués dans la régulation de la gìcogénolyse post mortem (BRISKeY et WISMERPEDERSEN, I95I; BENDALI, I955; KRZY WICKI et RATCLIFF, I 967), il n'en demeure pas moins que nos connaissances dans ce domaine restent très fragmentaires. Aussi nous a-t-il semblé opportun de procéder à différents temps post mortem à l'analyse systématique des différents substrats et des cofacteurs qui interviennent dans la transformation du glycogène en acide lactique. Selon notre opinion, une telle étude devrait permettre en effet d'accéder à une meilleure compréhension du mécanisme de la régulation de la glycogénolyse musculaire post mortem.

\section{MATÉRIEL ET MÉTHODES (1)}

Vingt porcs de race Large White et 20 porcs de race Piétrain constituaient le matériel expérimen. tal utilisé lors de cette étude. Le poids de ces animaux était compris entre roo et i ro kg. Les animaux de race Large White provenaient du troupeau expérimental de la Station de Recherches sur l'Élevage des l'orcs au $C . N . R$. Z. Ceux de la race Piétrain étaient achetés dans les élevages privés. Les animaux étaient abattus sous électronarcose à l'issue d'une période de jeûne de $\mathbf{r} 2$ heures. En échantillon d'un poids de roo grammes environ était prélevé sur le muscle Longissimus dorsi sitôt la saignée terminée. Après détermination du $\mathrm{pH}$ de cet échantillon on procédait au prélèvement de quatre fractions.

I $^{\circ}$ Une fraction de 5 grammes environ était broyée dans $50 \mathrm{ml}$ d'acide perchlorique $0,6 \mathrm{~N}$. Après centrifugation à $600 \mathrm{~g}$ pendant $\mathrm{r} 0$ minutes, le surnageant était neutralisé avec de la potasse $5 \mathrm{~N}$. Le précipité de perchlorate de potassium était éliminé par filtration. Les déterminations des substrats intermédiaires de la glycogénolyse de l'acide lactique, de l'ATP et de l'ADP et du glucose étaient effectuées sur le filtrat.

$2^{\circ}$ Une fraction de 2 grammes était broyée dans $5 \mathrm{ml}$ d'acide trichloracétique ıo p. ıoo. Le surnageant obtenu après centrifugation à $500 \mathrm{~g}$ pendant Io minutes constituait l'extrait brut. Les fractions phosphate désignées sous les termes de phosphate o minute, phosphate 7 minutes et phosphate acidosoluble total étaient dosées après dilution adéquate d'une partie de cet extrait brut.

Le phosphate o minute est le phosphate dosé en milieu acide dans un extrait de tissu à la température ordinaire. Le phosphate 7 minutes est le phosphate libre dosé après hydrolyse d'un extrait de tissu pendant 7 minutes à $100^{\circ} \mathrm{C}$ en présence d'HCl N.

Le phosphate acidosoluble total est obtenu après minéralisation en présence d'un mélange oxydant, $\mathrm{H} \mathrm{SO}_{2}$ Io $\mathrm{N}$ et $\mathrm{H}_{4} \mathrm{O}_{2} 30$ p. Ioo.

Le phosphate minéral réel représente l'orthophosphate réel du tissu.

Une partie de l'extrait brut était immédiatement neutralisée avec de la soude $0,4 \mathrm{~N}$. La créatine totale et la créatine libre étaient dosées dans cet extrait neutralisé. La différence entre la créatine totale et la créatine libre permettait d'évaluer la phosphocréatine.

(1) Pour la simplification de l'exposé, nous utiliserons les abréviations suivantes :

Phosphate "o minute": $\mathrm{P}_{0}$; Phosphate " 7 minutes " $: \mathrm{P}_{7}$; Phosphocréatine : $\mathrm{P}^{\mathrm{C}}$; Glucose I phosphate : GlP; Glucose 6 phosphate: $\mathrm{G} 6 \mathrm{P}$; Fructose 6 phosphate : $\mathrm{F} 6 \mathrm{P}$; Fructose I-6 phosphate : Ii $1-6 \mathrm{P}$; adénosine triphosphate : ATP ; adénosine diphosphate : ADP. 
$3^{\mathrm{o}}$ Une fraction de 0,5 à I $\mathrm{g}$ destinée à la détermination du glycogène était également immédia tement prélevée dans un tube à centrifuger préalablement taré contenant $3 \mathrm{ml}$ de KOH $35 \mathrm{p}$. Ioo.

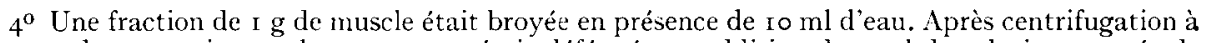
$500 \mathrm{~g}$ pendant 10 minutes, le surnageant était déféqué par addition de $2 \mathrm{ml}$ de solution saturée de baryte et $2 \mathrm{ml}$ de solution de $\mathrm{SO}_{4} \mathrm{Zn}$ Io p. Ioo faite extemporanément, de façon à neutraliser la solution de baryte volume à volume. Les sucres réducteurs totaux étaient dosés dans le filtrat.

Sitôt les diverses fractions prélevées, le reliquat de l'échantillon était placé dans un récipient immergé dans un bain marie à $30^{\circ} \mathrm{C}$ et dans lequel était maintenu un courant d'azote.

Les prélèvements étaient effectués ì différents temps après le début de l'incubation $(20,40,80$, [20, I 80 et 240 minutes) et les mêmes opérations étaient répétées.

Les muscles étaient classés en deux groupes en fonction de la valeur du $\mathrm{pH}$ après 40 minutes d'incubation. Lorsque le pH était supéricur à 6,00, la diminution du pH était considérée comme lente (groupe " $\mathrm{L} \|$ ) alors qu'elle était considérée comme rapide lorsque le pH était inférieur à 6,00 (groupe " $\mathrm{R})$ ).

Le groupe I comprenait 14 Large White et 4 Piétrains.

Le groupe $\mathrm{R}$ comprenait i6 Pietrains et 6 Large White.

Les $\mathrm{pH}$ " 40 minutes" des groupes $\mathrm{L}$ et $\mathrm{R}$ étaient respectivement de 6,20 $\pm 0,08$ et $5,55 \pm$ 0,1 2 .

Le $\mathrm{pH}$ était déterminé à l'aide d'un $\mathrm{pH}$ mètre EIL $23 \mathrm{AF}$.

Le glycogène était dosé selon la méthode de Good-Kramer SoMOGyi (I933). Nous avons modifié légèrement cette méthode en dosant le glucose final par la technique de IIUGUETT et NIXoN (I957) basée sur l'oxydation spécifique du glucose par la glucose oxydase et la détermination du peroxyde résultant à l'aide de la peroxydase en présence d'un chromogène accepteur d'oxygène. Les sucres réducteurs totaux étaient déterminés selon la méthode de FoLIN et WU (I920).

L'acide lactique, les substrats intermédiaires de la glycogénolyse, l'ATP, l'ADP étaient dosés selon la technique classique de BERGMEYER (1963).

Le principe général de ces méthodes consiste à apporter les substrats et enzymes nécessaires pour obtenir des réactions couplées de telle sorte qu'il apparaisse au terme de ces réactions un substrat sensible à l'action d'une déshydrogénase à nicotinamide nucléotide. L'addition d'un excès de cette déshydrogénase et du nicotinamide nucléotide déclenche alors la réaction. La quantité de substrat utilisée dans cette réaction est appréciée par la réduction ou l'oxydation du nicotinamide nucléotide que l'on mesure par la variation de densité optique à $340 \mu$. I.es substrats et enzymes utilisés dans cette étude provenaient des firmes Boehringer et Soehn et $\mathrm{XBC}$.

Le phosphate minéral était dosé selon la méthode de BerEnblum et Chain (1938).

Le phosphate o minute, 7 minutes et le phosphate acidosoluble total étaient dosés par la méthode de Fiske et SubBarow (1929).

La phosphocréatine était déterminée sclon la technique d'ENNOR-ROSENBERG (I952).

\section{RÉSULTATS}

\section{Evvolution des teneurs en glycogène, sucres réducteurs totaux et glucose (tabl. I)}

I a teneur en glycogène au temps zéro (c'est-à-dire cinq minutes après la fin de la saignée) présente une très grande variabilité puisqu'elle est comprise entre Io,o et $6 \mathrm{I}, \mathrm{I} \mu \mathrm{moles} / \mathrm{g}$. Les teneurs moyennes initiales sont respectivement de $40,3 \mu$ moles $/ \mathrm{g}$ pour le groupe $\mathrm{R}$ et de $44,6 \mu$ moles/g pour le groupe $\mathrm{L}$. Après une période d'incubation de 4 heures, ces teneurs sont de $0,5 \mu$ moles/g pour le groupe $R$ et de $30,4 \mu$ moles/g pour le groupe $\mathrm{L}$.

La teneur initiale en sucres réducteurs totaux exprimée en équivalents de glucose varie selon les animaux entre des valeurs extrêmes de 5,88 $\mu$ moles/g et 20,05 $\mu$ moles/g. Les valeurs moyennes figurant au tableau I n'ont qu'un caractère indicatif et comme le montrent les valeurs élevées des écarts-types, le mode d'évolution des sucres réducteurs totaux est très variable. 


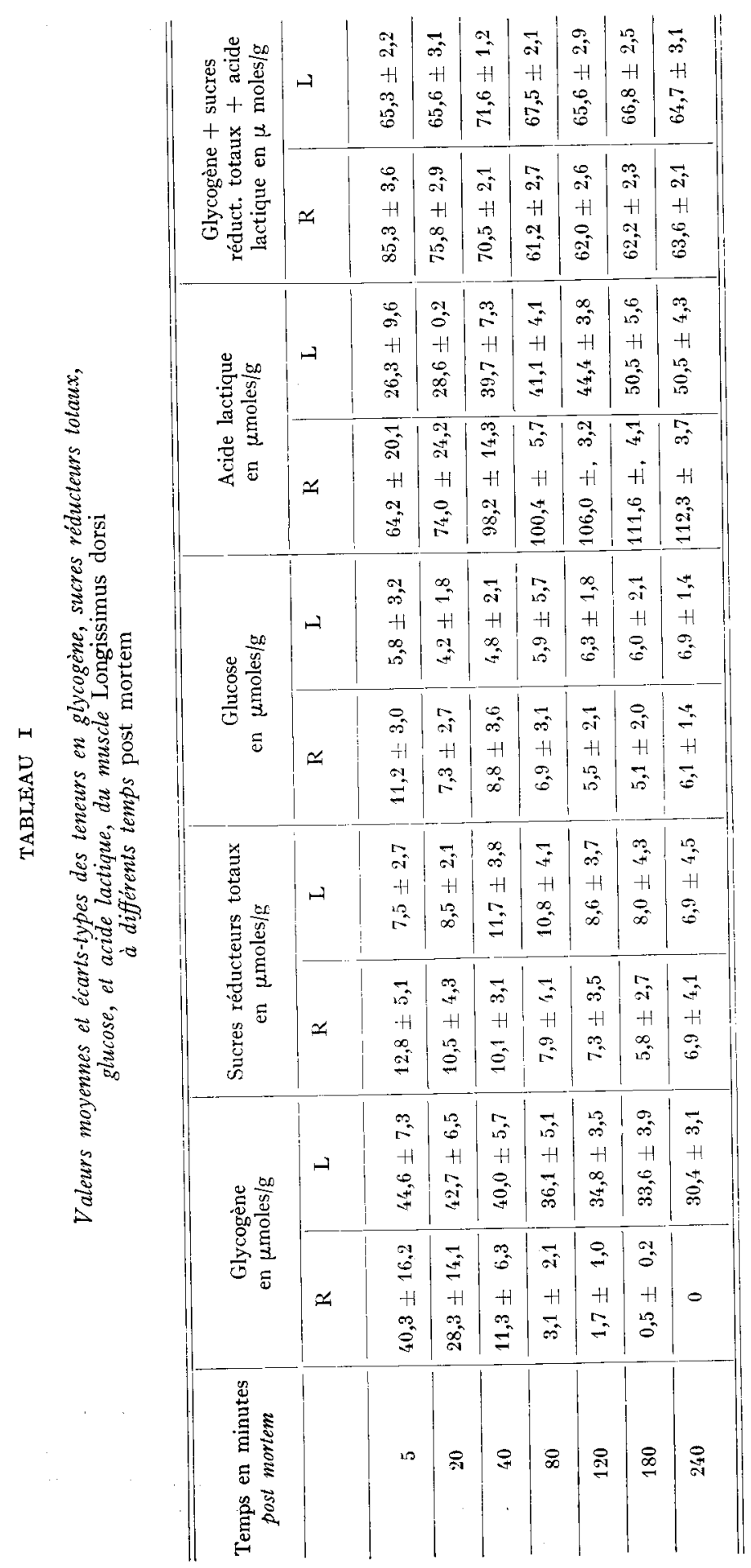


Néanmoins, on peut considérer que la teneur en sucres réducteurs totaux diminue dans un premier temps puis augmente ultérieurement pour atteindre des valeurs voisines de celles du temps zéro.

Les valeurs extrêmes des teneurs initiales en glucose sont de 4,22 et I2,55 $\mu$ moles/g. Ces valeurs sont inférieures à celles enregistrées pour les sucres réducteurs ce qui est logique puisque ces derniers englobent à la fois le glucose et divers intermédiaires de la glycogénolyse tels que le G6P, le F6P, le FI-6P. Dans la totalité des cas, la teneur en glucose diminue au début de l'incubation. Cette phase de diminution est alors suivie ou non d'une remontée du taux de glucose qui peut alors éventuellement atteindre et même dépasser les valeurs initiales.

\section{Evolution de la teneur en acide lactique (tabl. I)}

La teneur initiale en acide lactique présente une très importante variabilité puisque dans des conditions de prélèvements rigoureusement identiques nous avons pu constater des valeurs extrêmes de 16 et $88 \mu$ moles $/ g$. Il convient de remarquer que les valeurs initiales du groupe $R$ sont systématiquement supérieures à celles du groupe $L$. L'augmentation de la teneur en acide lactique se produit à des vitesses très variables suivant les individus. Jans certains cas, la valeur ultime est atteinte au bout de 40 minutes. Après 4 heures d'incubation, les taux d'acide lactique varient entre $4 \mathrm{I} \mu$ moles $/ \mathrm{g}$ et $\mathrm{I} 34 \mu$ moles $/ \mathrm{g}$. L'augmentation de la teneur en acide lactique, pendant cette même période, est comprise selon les échantillons entre 24 et $73 \mu$ moles/g.

\section{3. Évolution des teneurs en substrats intermédiaires de la glycogénolyse (tab1. 2)}

Les méthodes de dosage enzymatique que nous avons utilisées pour doser les substrats intermédiaires de la glycogénolyse ne permettent de déterminer que le G6P, le F6P, le FI-6P et 1'acide pyruvique. Le GIP est toujours présent en très faible quantité (inférieure à $0,3 \mu$ mole/g). Le taux de dihydroxyacétone est compris entre 0,3 et $0,6 \mu$ mole/g. Quant aux teneurs en glycéraldéhyde 3 phosphate, acide I-3 diphosphoglycérique, acide phosphoglycérique, acide I-3 diphosphoglycérique, acide 3 phosphoglycérique, acide 2 phosphoglycérique et acide phosphoénolpyruvique elles sont systématiquement inférieures à 0 , I $\mu$ mole/g, ce qui correspond en fait à la limite de détection des méthodes utilisées.

Nous ne considérerons donc que l'évolution du G6P, du F6P, du FI-6P et de l'acide pyruvique.

La teneur en G6P est relativement variable puisqu'elle est comprise entre 4,o et Io,8 $\mu$ moles/g. Les teneurs initiales sont plus élevées dans le cas des muscles à glycogénolyse rapide. Pendant les deux premières heures environ, le taux de G6P diminue dans la totalité des cas puis demeure constant ou bien augmente pour atteindre parfois des valeurs supérieures aux valeurs initiales. L'évolution du G6P est parallèle dans le cas des muscles à glycogénolyse rapide ou lente. Toutefois, les teneurs en G6P des muscles à glycogénolyse rapide restent constamment supérieures pendant toute la durée de l'incubation.

Les teneurs initiales moyennes en F6P des groupes $\mathrm{R}$ et $\mathrm{L}$ sont semblables. Les valeurs extrêmes sont de 0,03 et $2,36 \mu$ moles $/ g$. La teneur en F6P diminue pendant la 


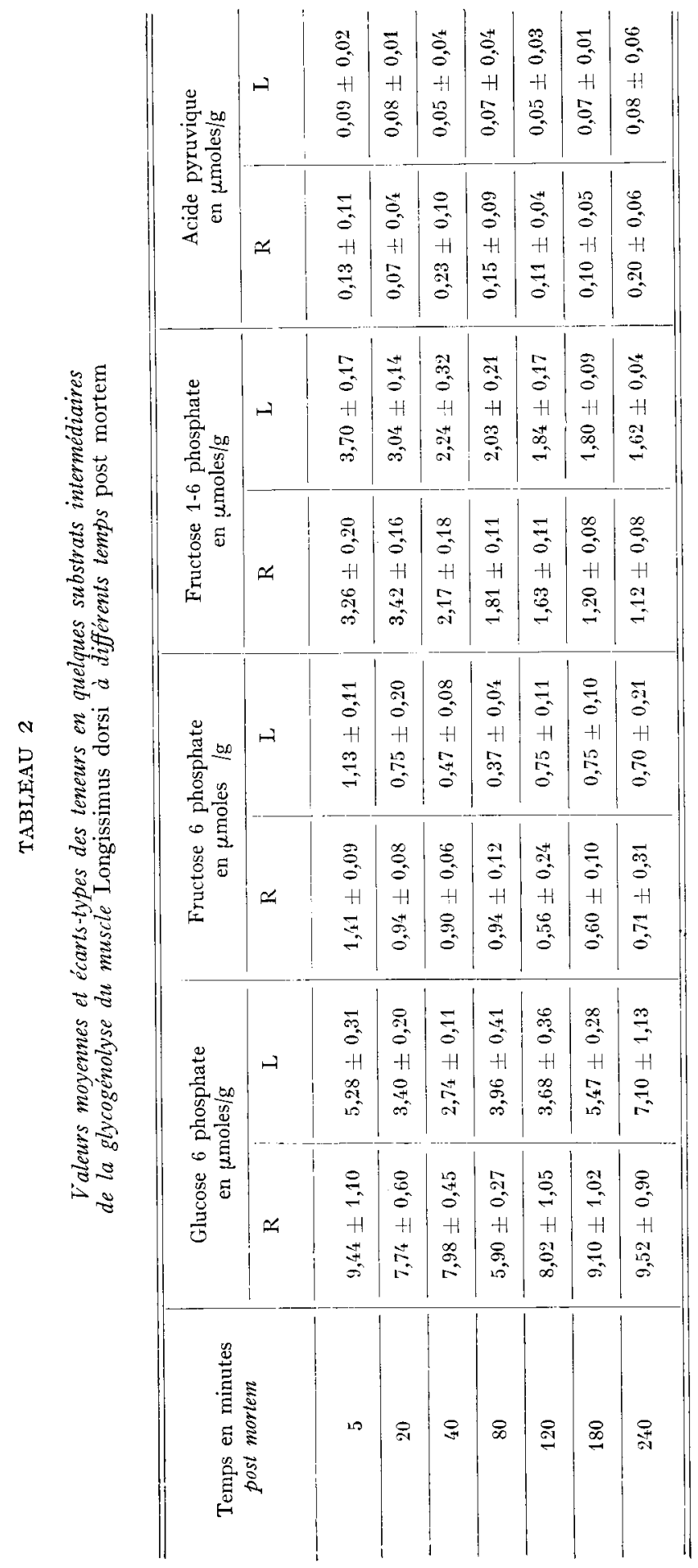




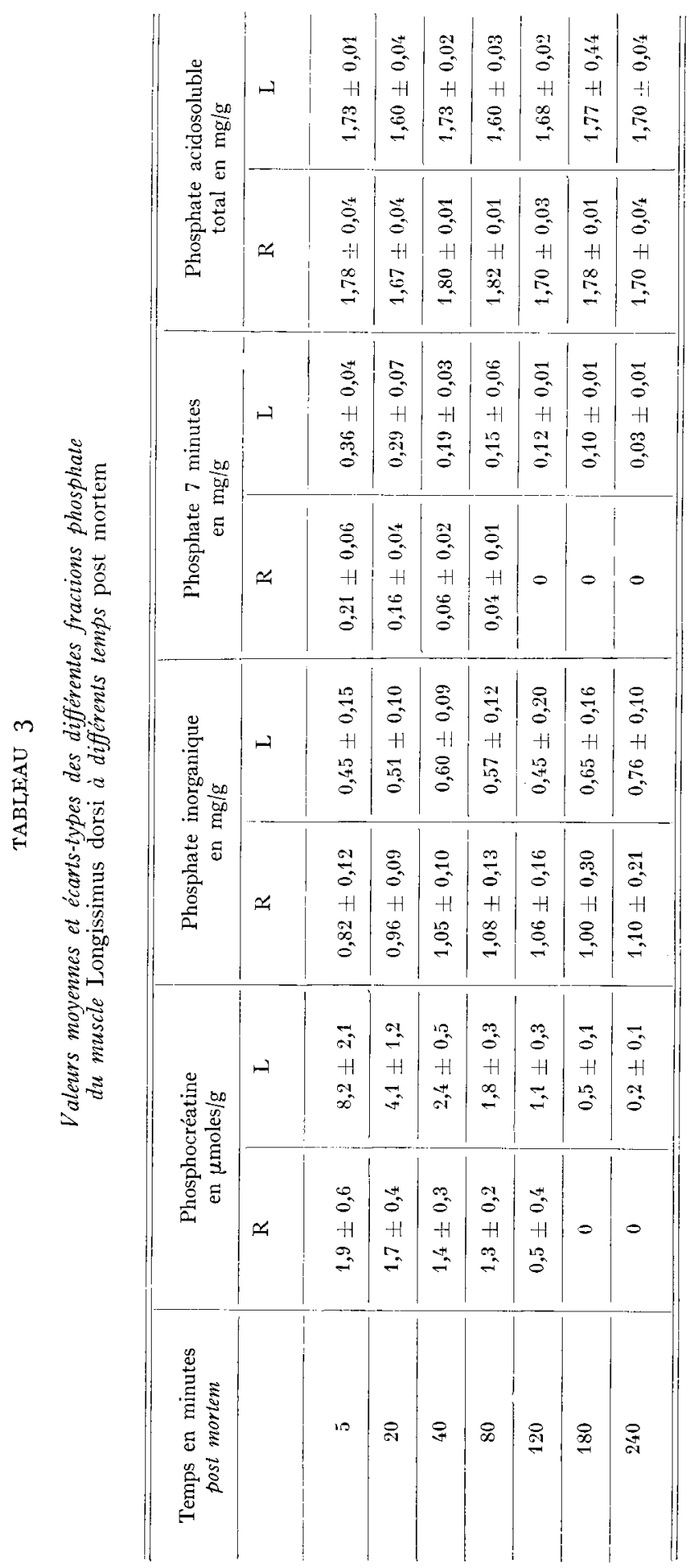


glycogénolyse, mais ne descend pratiquement jamais au-dessous de $0,4 \mu$ mole/g. Comme l'indiquent les résultats du tableau 2, l'évolution du F6P est semblable pour les muscles des groupes $L$ et $R$.

La teneur moyenne initiale en Fr-6P du groupe R est de 3,26 $\mu$ moles/g, et celle du groupe $L_{1}$ est de $3,70 \mu$ moles/g. L'évolution du FI-6P semble indépendante de la rapidité de la glycogénolyse. Le taux de Fr-6P diminue progressivement dans tous les cas pour atteindre des valeurs comprises généralement entre I et $2 \mu \mathrm{moles} / \mathrm{g}$.

Malgré des variations certes importantes, la teneur en acide pyruvique demeure néanmoins faible pendant toute la durée de la glycogénolyse et, bien que la quantité d'acide lactique augmente considérablement, il ne se produit simultanément aucune accumulation de son précurseur immédiat. Les taux d'acide pyruvique sont pratiquement semblables pour les muscles des groupes $\mathrm{L}$ et $\mathrm{R}$. L'analyse des fluctuations des teneurs en acide pyruvique en fonction de la vitesse d'apparition de l'acide lactique montre que le taux d'acide pyruvique tend à croître très légèrement lorsque la teneur en acide lactique augmente très rapidement.

\section{4. Évolution des diverses fractions phosphate, de la phosphocréatine, de l'ATP et de l'ADP (tabl. 3 et 4 )}

Le phosphate acidosoluble total initial est relativement constant et ne varie pratiquement pas pendant la glycogénolyse. Son taux moyen est de I,78 mg/g.

TABIEAU 4

Valeurs moyennes et écarts-types des teneurs en $A T P$ et $A D P$ du muscle Longissimus dorsi à diffírents temps post mortem

\begin{tabular}{|c|c|c|c|c|}
\hline \multirow{2}{*}{$\begin{array}{c}\text { Temps en minutes } \\
\text { post morlem }\end{array}$} & \multicolumn{2}{|c|}{ ADP en $\mu$ moles/g } & \multicolumn{2}{|c|}{ ATP en $\mu$ moles $/ \mathrm{g}$} \\
\hline & $\mathrm{R}$ & I. & $\mathrm{R}$ & $\mathrm{L}$ \\
\hline j) & $0,46=0,06$ & $0,40-0,11$ & $4,20 \div 0,80$ & $4,38 \pm 0,90$ \\
\hline 20 & $0,35 \pm 0,08$ & $0,20 ! 0,09$ & $2,11 \pm 0,70$ & $4,36 \pm 0,60$ \\
\hline 40 & $0,28 \pm 0,02$ & $0,20 \pm 0,09$ & $1,97 \pm 0,09$ & $3,62 \pm 0,30$ \\
\hline 80 & $0,25 \pm 0,09$ & $0,16: 0,10$ & $0,60 \pm 0,08$ & $2,85 \pm 0,25$ \\
\hline 120 & $0,26+00,09$ & $0,16 \pm 0,11$ & $0,07 \pm 0,03$ & $1,67 \pm 0,32$ \\
\hline 180 & $0,20 \pm 0,05$ & $0,10-0,07$ & 0 & $0,45 \pm 0,01$ \\
\hline 210 & $0,19-0,07$ & $0,19 \pm 0,05$ & 0 & 0 \\
\hline
\end{tabular}

Par contre, la teneur en phosphate minéral s'avère très variable. Les muscles présentant une glycogénolyse particulièrement rapide ont, en effet, une teneur en phosphate minéral systématiquement plus élevée. Celle-ci est de $0,82 \mathrm{mg} / \mathrm{g}$ alors qu'elle est en moyenne de $0,45 \mathrm{mg} / \mathrm{g}$ dans le cas de muscles à glycogénolyse lente. 
Les résultats figurant au tableau 3 montrent que la teneur en phosphate inorganique augmente au cours de la glycogénolyse. Ces données relatives à des valeurs moyennes ne traduisent toutefois que d'une façon approximative le mode d'évolution de la teneur en phosphate inorganique qui présente en fait une très grande variabilité. C'est ainsi que, selon les échantillons, la quantité de phosphate inorganique peut augmenter d'une façon régulière ou bien présenter des phases alternatives de diminution puis d'augmentation.

La teneur initiale en $\mathrm{P}_{7}$ est comprise entre $0, \mathrm{I} 6$ et $0,46 \mathrm{mg} / \mathrm{g}$. Les valeurs moyennes pour les groupes $L_{\text {, et }} \mathrm{R}$ sont respectivement de $0,36 \mathrm{mg} / \mathrm{g}$ et de $0,2 \mathrm{I} \mathrm{mg} / \mathrm{g}$. I a diminution du taux de $\mathrm{P}_{7}$ est plus rapide dans les muscles du groupe $\mathrm{R}$. Dans de nombreux cas on assiste à une déplétion quasi totale du $\mathrm{P}_{7}$ après 4 heures d'incubation.

Les teneurs en phosphocréatine des muscles des groupes $\mathrm{R}$ et $\mathrm{L}$, diffèrent notablement comme l'indique le tableau 3. Pour le groupe $\mathrm{L}$ la valeur moyenne est en effet de 8,2 umoles/g alors que pour le groupe $\mathrm{R}$ elle n'est que de $I, 9 \mu \mathrm{mole} / \mathrm{g}$. Dans certains cas d'ailleurs, compte tenu de la précision de la méthode utilisée, il est pratiquement impossible de détecter la présence de phosphocréatine. I a déplétion de la phosphocréatine est enfin beaucoup plus rapide dans les muscles du groupe $R$.

Le taux initial d'A'TP présente des variations relativement importantes. Les valeurs extrêmes sont en effet de I,9 et 5,6 $\mu$ moles/g. I a teneur moyenne d'ATP des muscles du groupe R est de 4,20 $\mu$ moles/g, et celui du groupe $\mathrm{L}$, de $4,38 \mu$ moles/g.

Les muscles du groupe $L$ se caractérisent par une persistance du taux initial d'ATP ou éventuellement par une très lente diminution pendant la première heure d'incubation. Dans les muscles du groupe R, la chute du taux d'A'TP est beaucoup plus rapide. Dans le cas des glycogénolyses rapides, 1'ATP est pratiquement disparu au bout de 3 heures d'incubation.

La quantité d'ADP présente dans le muscle sitôt le prélèvement, est relativement constante $(0,46$ et 0,40 umole/g respectivement pour les groupes $R$ et $\mathrm{L})$. Le taux d'ADP diminue rapidement dans les 20 premières minutes pour se stabiliser à des valeurs comprises entre 0,1 et 0,3 mole/g.

\section{DISCUSSION}

L'ensemble des résultats que nous venons de rapporter montre que, lorsque la glycogénolyse anaérobie est particulièrement rapide, le muscle présente un certain nombre de caractéristiques dont il convient de faire la synthèse pour interpréter le mécanisme de la régulation de la glycogénolyse post mortem.

Tout d'abord, compte tenu des fortes teneurs initiales en acide lactique et, simultanément, des valeurs très faibles dela teneur en glycogène de certains échantillons, il apparaît nettement qu'immédiatement avant le prélèvement ou peut-être plus vraisemblablement dans les minutes qui suivent celui-ci, la glycogénolyse peut être exacerbée à un point tel que la majeure partie du glycogène est déjà transformée en acide lactique. Ia confrontation des résultats concernant les teneurs en glycogène et en acide lactique des mêmes échantillons montre que les faibles valeurs du taux initial de glycogène sont toujours associées à des teneurs en acide lactique plus élevées, ce qui indique d'une part que la glycogénolyse s'est produite instantanément et, d'autre part, 
que la quantité de glycogène présente effectivement immédiatement avant ou lors du prélèvement ne pouvait en aucun cas constituer un facteur limitant pour un déroulement normal pour la glycogénolyse. Il est vraisemblable que des différences structurales du glycogène peuvent contribuer à expliquer cette activité glycogénolytique particulièrement rapide, qu'il s'agisse d'une modification du degré de ramification et de la longueur des chaînes (LAwrIE et al., I959; BRISKEY et HOEKSTRA, I963) ou bien de l'importance relative des formes " libres » et « liées » comme nous l'avons montré antérieurement (ChARPENTIER, I963) confirmant en cela les observations de WISMERPEDERSEN et Briskey ( I96r). Les teneurs initiales en sucres réducteurs totaux que nous avons observées sont du même ordre que celles rapportées par BoDWEL, et al. (Ig66). Selon ces auteurs, les teneurs moyennes en sucres réducteurs totaux sont de $12,6 \pm$ $3,7 \mu$ moles/g pour les muscles à glycogénolyse rapide et de I I, $6 \pm$ I, $5 \mu$ moles $/ g$ pour les muscles à glycogénolyse lente alors que nous obtenons des valeurs respectivement de I $2,8 \mu$ moles $/ g$ et $7,5 \mu$ moles $/ g$. Ces valeurs sont inférieures à celles rapportées par WISTER-PEDERSEN (I959) pour le muscle Longissimus dorsi des porcs Landrace. Elles sont, par contre, supérieures au taux de $7,4 \mu$ moles/g établi d'après les données de SHARP (1962) pour le muscle Longissimus dorsi également.

Bien que dans la littérature les sucres réducteurs totaux reçoivent souvent l'appellation fallacieuse de glucose, il convient de faire la discrimination entre le glucose réel du tissu musculaire déterminé par des méthodes spécifiques et le "glucose " dosé par les méthodes utilisant le pouvoir réducteur de divers glucides, dont, entre autres, le glucose. La comparaison du taux de sucres réducteurs totaux déterminé par la méthode de Folın-Wu et du taux de glucose déterminé par la méthode à la glucose oxydase montre que la différence entre les deux varie de $0,1 \mathrm{mg} / \mathrm{g}$ à $\mathrm{I}, 9 \mathrm{mg} / \mathrm{g}$.

Cette différence correspond pratiquement à la somme de G6P et du FI-6P qui sont les deux principaux substrats intermédiaires de la glycogénolyse.

L'évolution du glucose au cours de l'incubation en anaérobiose indique, qu'au début du moins, le glucose est transformé en acide lactique. Le taux de glucose remonte après 20 minutes environ par suite vraisemblablement d'une dépolymérisation du glycogène résiduel due à l'action conjuguée de l'amylase, de 1'amylo I-6 glycosidase et de la maltase présentes dans le tissu musculaire (SHARP, I962).

Comme nous l'avons précédemment montré, la différence entre la teneur en sucres réducteurs totaux et la teneur en glucose s'explique principalement par la présence de G6P. Cet intermédiaire est d'ailleurs le seul qui soit susceptible de s'accumuler au cours de la glycogénolyse. Comme l'indique la figure 2, le G6P continue à augmenter alors que la teneur en acide lactique atteint un palier. Cette augmentation du taux de G6P résulte de l'action inhibitrice du $\mathrm{pH}$ sur la phosphohexose isomérase et la phosphofructokinase. La présence de G6P en plus grande quantité dans les muscles à glycogénolyse rapide indique que la phosporylation du glycogène est plus active dans les muscles de ce type. Il est vraisemblable qu'une décharge d'adrénaline immédiatement avant l'abattage est à l'origine de cette phosphorylation plus active. Celle-ci implique obligatoirement une consommation d'ATP pour la transformation de la phosphorylase $b$ en phosphorylase $a$.

Une phosphorylation plus intense du glycogène tend donc à diminuer le taux d'ATP. Or, nous observons précisément que les muscles à teneur élevée en G6P présentent une faible teneur en A'TP. 
L a transformation du glycozène ne se produit que très rarement d'une façon stœchiométrique. Dans certains cas, il semble en être ainsi si l'on considère le bilan global mais en fait l'étude cinétique montre que le plus souvent au début de la glycogénolyse la quantité d'acide lactique formé est supérieure à la quantité de glycogène disparu alors qu'ensuite c'est l'inverse qui se produit. Une partie de l'acide lactique est en effet synthétisée au début à partir du glucose et du G6P, qu'ensuite une fraction du glycogène résiduel est transformée vraisemblablement en dextrines et glucose.

Si nous considérons la somme du glycogène, des sucres réducteurs totaux et de l'acide lactique, nous constatons que cette somme demeure pratiquement constante pendant la durée de la glycogénolyse dans le cas des muscles du groupe $\mathrm{L}$, mais diminue, par contre, dans le cas des muscles du groupe $\mathrm{R}$ (tabl. I).

L'absence de relation stœchiométrique dans ce cas laisse supposer qu'un produit du catabolisme du glycogène ou du glucose non identifié, s'accumule lorsque la glycogénolyse est rapide.

Il convient de remarquer également que la somme globale du glycogène, des sucres réducteurs totaux et de l'acide lactique est plus importante dans le cas des muscles à glycogénolyse rapide, ce qui corrobore les résultats de BODWEL, et PEARSON (I966).

Comme nous l'avons mis en évidence, mis à part le cas du G6P, il ne se produit pas d'accumulation de substrats intermédiaires de la glycogénolyse et les teneurs de ces substrats ne présentent pas de variations significatives en fonction de la vitesse de la glycogénolyse.

Il ne semble donc pas que les modifications d'activités des enzymes impliquées dans la série de réactions de la glycogénolyse puissent, en fait, provoquer la très grande variabilité que l'on constate dans les vitesses de glycogénolyse musculaire post mortem.

Il est bien établi que la nature de l'équipement enzymatique des muscles peut effectivement varier en fonction de leur orientation métabolique (DAwson et al., I964) et que notamment l'importance relative des isoenzymes de la lactodéshydrogénase est différente selon qu'il s'agit de muscles glycogénolyse lente ou rapide (CHARPENTIER et GOUTEFONGEA, I964). Ces différences sont, semble-t-il, à rapprocher de celles qui existent entre les tissus dont le métabolisme est orienté préférentiellement soit vers l'aérobiose, soit vers l'anaérobiose.

Compte tenu néanmoins des remarquables potentialités d'accélération de la glycogénolyse musculaire in vivo, il ne semble guère concevable d'envisager que des modifications d'activités des enzymes de la glycogénolyse soient à l'origine des différences constatées dans la rapidité de celle-ci post mortem (BENDALL, I965).

$\mathrm{L}$ a variabilité des taux de $\mathrm{PC}$ que nous avons constatée correspond tout à fait à celle rapportée par différents auteurs (BENDALL et al., I963; BoDwELL et al., I965). L'absence de $\mathrm{PC}$ dans certains cas confirme les résultats antérieurs de BoDWELL (I965). La valeur initiale plus faible du taux de PC et sa disparition plus rapide dans les muscles à glycogénolyse rapide confirme également les résultats de BENDALI, et al., (I963). En ce qui concerne le taux initial d'ATP, il convient de remarquer que sa variabilité est relativement faible. Néanmoins, lorsque les valeurs du taux de $\mathrm{PC}$ sont très basses, la teneur initiale en ATP peut atteindre fréquemment les valeurs de l'ordre de 2 à $3 \mu$ moles/g, alors que les valeurs les plus courantes sont comprises entre 5.5 et 
$6,5 \mu$ moles/g. Ces valeurs correspondent à celles rapportées par BoDwELL et al. (1965) mais sont, par contre, inférieures à celles citées par BENDALI, et al., (I963).

A ce propos, il convient de noter que de nombreux auteurs déterminent le $\mathrm{P} 7$ pour estimer la teneur en ATP, or nos résultats montrent une nette discordance entre les valeurs du taux d'ATP et de celui du $\mathrm{P}_{7}$, ce qui confirme d'ailleurs pleinement les observations de BoDWeli et PEARson (I965). La chute du taux d'ATP est beaucoup plus rapide dans le cas des muscles à glycogénolyse rapide. Dans les cas extrêmes, la déplétion peut être totale au bout de I heure d'incubation. En tenant compte des teneurs initiales et finales en ATP, PC et glycogène, il est possible de calculer l'activité ATPasique du tissu musculaire. Un tel calcul nous conduit à des activités ATPasiques respectivement de 0,16 umole d'ATP dégradé/g/minute dans le cas des muscles du groupe $\mathrm{L}$ et $0,3^{8} \mu$ mole d'ATP dégradé $/ \mathrm{g} /$ minute dans le cas des muscles du groupe $\mathrm{R}$ pendant les I 20 premières minutes. La principale différence entre les muscles présentant des vitesses de glycogénolyse plus ou moins rapides se situe en définitive au niveau de leur activité ATPasique. Le mécanisme de la régulation de l'activité A'TPasique post mortem n'est pas encore élucidé. Les ATPases du muscle peuvent se classer en trois catégories : l'actomyosine ATPase dont l'activité dépend de la concentration en calcium libre, 1'ATPase mitochondriale et la ou les A'TPases du réticulum sarcoplasmiques activées par le sodium et le potassium. Selon BENDALI. (I96I), la chute de l'ATP post mortem serait due vraisemblablement à l'action des ATPases sarcoplasmiques mais la démonstration directe de leur action n'a toutefois pu être effectuée. Il est bien établi que l'activité A'TPasique des microsomes des muscles blancs est supérieure à celle des muscles rouges (GERGEI,Y, I965). Comme l'ont montré par ailleurs les travaux de KRZYWICKI et RATCI,IFF (I967), il existe en outre une relation inverse entre la teneur en phospholipides du muscle de porc et l'intensité de la glycogénolyse anaérobie. Il semble donc concevable de supposer que des muscles présentant une glycogénolyse post mortem particulièrement rapide se caractérisaient éventuellement par une importance relative plus grande du réticulum sarcoplasmique et par là même auraient une activité ATPasique supérieure. Il ne s'agit toutefois là que d'une hypothèse dont la validité mériterait d'être vérifiée au cours d'expériences ultérieures.

Une des conséquences immédiates de l'exacerbation de l'activité ATPasique est de provoquer une augmentation du taux de phosphore inorganique. Or les travaux de Morgan et PARMEGgiani ont montré nettement que la quantité de phosphore inorganique intracellulaire était responsable de la régulation de la glycogénolyse.

Les modalités d'intervention du phosphore inorganique dans la régulation de la glycogénolyse sont en effet multiples. Tout d'abord le phosphore inorganique intervient en tant que substrat au niveau de la phosphorylation du glycogène et de l'oxydation des trioses phosphate. Il exerce aussi un effet allostérique d'activation sur la phosphofructokinase (LARDYZ, I965). ROSE (I965) a montré en outre que le phosphore inorganique augmente le $\mathrm{Km}$ de l'aldolase pour l'hexosediphosphate, ce qui entraîne une activation de la phosphofructokinase car cet enzyme est activé par le produit de la réaction. La diminution concomitante du taux d'ATP annule d'ailleurs simultanément l'inhibition de la phosphöructokinase (PASsONNEAU et LAwRY, I962). Notons également que la diminution du taux d'ATP provoque une activation de la phosphorylase dans le tissu anoxique (Morgan et PARMEGGrani) et contribue aussi à l'activation de la phosphorylation du glycogène. L'ADP enfin intervient comme 
substrat dans la glycogénolyse et il est donc logique que celle-ci soit plus intense si la quantité instantanée d'ADP disponible est plus élevée.

La synthèse des diverses observations effectuées à l'occasion de cette étude nous permet de dégager quelques conclusions quant aux facteurs responsables de l'accélération de la glycogénolyse post mortem. L'hypothèse selon laquelle une ou plusieurs réactions " limitantes" de la chaîne glycolytique seraient activées ne peut être valablement retenue. La faible variabilité des substrats intermédiaires permet en effet d'éliminer l'éventualité d'un dérèglement enzymatique à ce niveau. Tout au plus l'activation de la réaction initiale, c'est-à-dire la phosphorylation du glycogène, peut être invoquée. Cette relative constance des substrats intermédiaires indépendamment de la rapidité de synthèse de l'acide lactique, ne saurait en fait nous surprendre si l'on considère que la glycogénolyse constitue un remarquable système intégré dans lequel les diverses réactions sont synchronisées selon un mécanisme dont les rouages ne sont pas encore parfaitement connus. Les travaux de KARPATKIN et al. ont montré que lors de stimulations électriques répétées, les concentrations de substrats intermédiaires n'étaient pas modifiées alors que le flux glycolytique était multiplié par 30. Dans le cas du muscle de porc post mortem, ce facteur dans les cas extrêmes n'est que de 5 . Il n'est donc pas surprenant que les teneurs en substrats intermédiaires soient relativement peu modifiés. La caractéristique essentielle en définitive des muscles à glycogénolyse rapide consiste en l'exacerbation de leur activité A'TPasique. Une activité ATPasique accrue entraînant par conséquent une augmentation du taux de phosphore inorganique, semble, en fait, être à l'origine de l'accélération de la glycogénolyse. Le problème essentiel consiste donc à élucider les mécanismes responsables de la rézulation de l'activité ATPasique post mortem. Il est indispensable de connaître au préalable la part relative des diverses ATPases du muscle dans l'activité ATPasique globale du tissu musculaire post mortem et de préciser également les modalités de leur action.

Rę̧u pour publication en juin 1968.

\section{SUMMARY}

BREAKDOWN OF GLYCOGEN « POST MORTEM »

IN THE " LONGISSIMUS DORSI" MUSCLE OF PIGS

It has been well established that the rate of fall in $\mathrm{pH}$ in muscle of pigs post mortem is very variable and that it directly influences many aspects of the quality of the meat. Therefore the present study consists of a descriptive study of the breakdown of glycogen post mortem in pig muscle in order to explain if possible the differences in speed of this breakdown. The study was of 20 Large White and 20 Piétrain pigs of weights between 100 and $\mathrm{I} 10 \mathrm{~kg}$.

The estimations were made periodically on a portion of the longissimus dorsi taken immediately after slaughter and kept under nitrogen at $30^{\circ} \mathrm{C}$ for 240 minutes. The muscles were classed in 2 groups according to $\mathrm{pH}$ after 40 minutes. When the value was above 6.00 the reduction in $\mathrm{pH}$ was considered slow (Group L) and when pH was below 6.00 the reduction was considered rapid (Group R). In group L there were 14 Large White and 4 Piétrain and in group $\mathrm{R}$ there were 16 Piétrain and 6 Large White.

Estimations were made of glycogen, glucose, total reducing sugars, lactic acid, intermediate substrates of the breakdown of glycogen, adenosine triphosphate (ATP), adenosine diphosphate (ADP). phosphocreatine, mineral phosphate, total acid-soluble phosphate, $\mathrm{P}$ ( $\circ$ minutes » and $\mathrm{P}$ " 7 minutes», 
The results of this study show difference in initial contents as regards principally phosphocreatine (R t.9 umoles per g; L 8.2 $\mu$ moles per g), mineral phosphate (R o.82 $\mu$ mole per g; I. o.45 umole per g) and lactic acid ( $\mathrm{R} 46.2 \mu$ moles per $\mathrm{g} ; \mathrm{I}, 26.3 \mu$ moles per $\mathrm{g}$ ). The small variability in intermediary substrates suggests that difference in activity of enzymes concerned in the breakdown of glycogen does not seem to be the cause of difference in rate of breakdown post mortem. The main difference between groups $\mathrm{R}$ and $\mathrm{L}$ was in ATP-ase activity. The actual ATP-ase activity was on average $0.16 \mu$ mole ATP degraded per gram per minute for group L and $0.38 \mu$ mole for group R. The essential problem thus consists of elucidating the mechanisms concerned in the regulation of ATP-ase activity post mortem.

\section{RÉFÉRENCES BIBLIOGRAPHIQUES}

Bendali. J. R., ig6i. Post mortem changes in muscle. In, Structure and linuclion of muscle, 227-274, cd. by G. H. Bourne. Academic: P'ress.

Bendall. J. R., Haliund O.. Wisnigr-PEDersen J., 1,62. A study of the pest mertem pll changes in the muscle of Danish Landrace pigs in relation to the oc'curence of watery pork. Sth lleeting of Meal Research Workers. Mosicou.

Bendall J. R., Halidind O., Wismer-Pedersen J., 1963 . Post mortem changes in the muscles of Iandrace pigs. J. Food Sci. 23, $156-162$.

BENDALL J. R., $196_{5}$. The effect of pretreatment of pigs with curare on the post mortem rate of pH fall and onset of rigor moris in the musculature. 11th European. Heeting of lleat Research Workers. Belgrade.

Berenblum I., Cimain E., 1938. An improved method for colorimetric determination of phosphate. Biochem. J., 32, 295-300.

Bergmeyer H. U., ig63. Methods of enzyme analysis. Academic Press N. Y. 1963.

Bodwell C. E., Pearson A. M., Spooner M., i965. Post mortem changes in muscle. I. Chemical changes in beef. J. Food Sci., 30, $766-772$.

Bodwell C. E., Pearson A. M., Wisuer-Pedersen J., Bratzler L. J., ig66. Post mortem changes in muscle. II. Chemical and physical changes in pork. J. Food Sci., 31, I-12.

BRISkEY F;. J., Wismer-PEDERSEN J., Ig6r. Biochemistry of pork muscle structure. I. Rate of anaerobic glycolysis and temperature change versus the apparent structure of muscle tissue. J. Food Sci., 26, $297-305$.

Charpentier J., Gouterongea R., 196.4. Electrophoretic characteristics of lactic dehydrogenase in normal and exudative pig muscle. Valure, $201,13^{2} 5^{-1} 3^{26}$.

Charpentier J., 1966. Relation entre la teneur du muscle de porc en desmo- et lyoglycogenése post mortem. Ann. Zoolech., 15, 367-371.

Dawson I. M., GoOdFriend T. L., Kal'LAN N. O., I964. Lactic dehydrogenases functions of the two types. Science. 143, 929-933.

ENNOR A. H., Rosenberg; II., 1952. 'The deternination and distribution of phosphocreatine in animal tissues. Biochem. J., 51, 606-510.

Fiske G. H., Subbarow I., I929. Phosphocreatine. J. Biol. Chem., 81, 629-679.

Folin A., Wu H., 1920. A simplified and improved method for determination of sugar. J. Biol. chem., 41, 367-375.

Gergely J., Pragay D., Scholz A. F., Seidel J. C., Sreter F. A., 'Iinompson M. M., ig65. Comparative studies on white and red muscle. In : Molecular Biologv of muscular contraction, Ed.S. EBASHI. EIsevier publishing company, $145^{-1} 59$.

Good C. A., Kramer H., Somogyi A., 1933. The determination of glycogren. J. Biol. Chem., 100, 485491 .

HuguetT A. S., Nixon D. A., 1957. Einzymatic determination of blood glucose. Biochem. J., 66, 12 p.

Karpatkin S., Helmreich li., Cori C. F., I964. Regulation of glycogenolysis in muscle. Effect of stimulation and epinephrine in isolated frog sartorius muscle. J. Biol. Chem., 289, 31 39-3145.

Kastenchmidt L. L., Briskey E. J., IIokkstra W. G., ig66. Metabolic intermediates in skeletal muscles with fast and slow rates of post mortem glycolysis. Nature, 212, 288-289.

Krzywicki K., Wismer-Pedersen P. J., 1962. Some properties of sarcoplasmic proteins of normal and watery pork muscle. 8th Meeting Meat Research Workers. Yoscou.

KRZYWICKI K., RATCLIFF P. W., I 967 . 'The phospholipids of pork muscle and their relation to the post mortem rate of glycolysis. J. Sci. Fd Agric., 18, 252-257.

LARDY H. A., I965. Regulation of energy yielding processus in muscle. In, The physiology and Biochemistry of muscle as a Food. edited by L. J. Brisker, R.-. Cassens, J. C. Trautuan. Lniv. Wisconsiu Press.

LAWRie R. A., Manners D. J., Wright A., I959. Alpha I-4 glucosans. Glycogen structure and rigor mortis in mammalian muscles. Biochem. J., 78, 485-490. 
LAWRIE R. A., 1960. Post mortem glycolysis in normal and exudative longissimus dorsi muscles of the pig in relation to so called white muscle disease. J. Comp. Pathol. Therap., 70, 273-295.

McLoughlin J. V., I963. The effect of rapid post mortem $\mathrm{pH}$ fall on the extraction of the sarcoplasmic and myofibrillar protein of post rigor muscle. Irish J. Agric. Res., 2, I I 5-I 24.

Morgan H. E., Parmeggiani A., I964. Regulation of glycogenolysis in muscle. In : Control of glycogen melabolism. Fds. J. A. Churchill Eid.

Passonneau J., Lowry O., 1962. Phosphofructokinase and the Pasteur effect. Biochem. Biophys. Res. Com., 7, IO-I5.

Penny I. F., I 967 . The influence of $\mathrm{pH}$ and temperature on the properties of myosin. Biochem. J., 104, $609-6$ r 5 .

Rose I. A., ig65 In B. Chance (ed). A symposium on control of Energy metabolism. Academic Press. New York.

Sayre R. N., Briskey E. J., Hoekstra W. G., 1963. Porcine muscle glycogen structure and its association with other muscle properties. Proc. Soc. Exptl. Biol. Med., 112, $223^{-225}$.

SHarp J. G., 1962. Non enzymic browning determination in dehydrated meat. In, Recent Advances in Food Science, 2, 65-78. Edited by J. HAwTHORN et J. MUIL LEITCH.

SCOPES R. K., r964. Solubilities of muscle proteins. Biochem. J., 91, 201-207.

Wismer-Pedersen J., I959. Quality of pork in relation to rate of pH change post mortem. Food Res., 2, 7 II-727.

WiSMER-PEDFRSEN J., BRISKEY L. J., I96Ia. Rate of anaerobic glycolysis versus structure in pork muscle. Nalure, 139, 318-320. 